\title{
A MODEL AND METHOD FOR SOLVING A DYNAMIC TRANSPORT PROBLEM
}

\author{
Gediminas Davulis \\ Department of Economic, Law University of Lithuania, Ateities pr. 20, LT-08303 Vilnius-57, Lithuania. \\ E-mail:ekk@ltu.lt
}

Received 1 December 2003; accepted 10 February 2004

\begin{abstract}
The problem of optimal development of transport network is considered. We have to define a plan of network development, i.e. a network state at fixed time moments possible the scope of allocated resources such that the total expenses for reconstruction of the network and construction of its new elements as well as for passenger and cargo transportation be the lowest. Thus the problem considered can be described by the optimization model with a non-linear nonconvex objective function and linear constraints of special structures. Since that is a non-convex problem with a lot of extreme therefore one can expect to find only an approximate solution, close to a global one, at best. There is no effective and universal solution methods for this problem even in the sense of a local solution. This paper discusses a method for solving the problem using the synthesis of static section, that allows us to decompose dynamic problem into the set of static problems of a smaller volume, and contour optimization methods. The experimental calculation confirm that the proposed method is suitable for solving problem represented in the paper.
\end{abstract}

Keywords: planning of network development, flow distribution, mixed-variables optimization problems

\section{Introduction}

The problem considered is formulated as a problem of optimal development of the transport network the solution of which determines the dynamics of multicommodity transport flows and network state variation in a given period. This problem essentially consists of two interrelated problems. The first one is design of the network structure and determination of the most rational state of its elements in each year of the considered period. The second problem is optimization of passenger and cargo flow distribution in the network. Constantly increasing volumes of passenger transportation require a constant transport network development as well as of renewal and updating of its elements, i.e., improvement of its technical state. In order to improve and update the transport infrastructure we need some financial, material, and other means the volume of which is limited. Therefore, of all the network extension variants we have to choose such that satisfies the needs for transport services best and can be realised with the available resources. Thus, the problem of long-term transport network development is an optimization problem characterized by great volumes, non-linear relations, one part of variables of which is continuous, while the other part is discrete.

At present quite a few methods are known for solving transport problems $[1,2]$. Though these problems are described by optimization models, a direct use of the known mathematical programming methods is not the proper way for solving transport problems. It is not suitable to solve problems even of small volume since the general methods do not estimate the problem specifity would be not economical and ineffective. On the other hand, even special methods oriented to solving problems of small volume are not always acceptable to solve practical problems that are characterized both by non-linear links and great volume, i.e., by a large number of network elements and transport flow kinds. Thus, the basic obstacle to be taken in solving optimal network development problems that are of importance in practice is their volume. Requirements to the methods used grow up respectively, they should be powerful enough to take this obstacle. The most successful ways for solving problems under consideration are those that are based 
on decompositional, heuristic principles, or on the use of interactive simulation modelling systems by arranging the solution process in an interactive mode of operation. If very great volume problems are considered or problem constraints express very complicated links relations among the problem variables, then most often it remains the heuristic way of solution which at best can ensure a good enough admissible solution only. Heuristic procedures, that allow for satisfactory solutions in an agreeable calculation time, are based on the following principles: simplification of a problem by ignoring a part of constraints or replacing them by simple ones, most frequently, by non-linear ones; decomposition of the initial problem into smaller partial problems ignoring their interrelations; identification of no good problem solution trends and their rejection; formation of an iterative process for improving the initial solution, and so on. Heuristic procedures have a drawback that the solution obtained can be far from optimal in the sense of both, the problem criterion and problem variables. A better result is achieved if heuristic procedures are used in interactive systems that operate in the dialog mode. In this case, dependent on the results obtained during solution, we are able to change the strategy of solution and even the model itself. Meanwhile, on a lower level of a system, particular calculations are performed relying on heuristic procedures.

Better results are obtained by using decomposition methods that allow for decomposition of the initial problem into a sequence of problems of smaller volume. As usual, a dynamic problem is decomposed first with respect to the time parameter, i.e., the initial network expansion problem is replaced by a sequence of static problems solved at fixed time moments. This kind of decomposition is natural in case the time parameter is a discrete variable. If certain conditions are satisfied, i.e., if flows in the transport network are not decreasing, then it suffices to solve a sequence of static problems, called static sections, only once [3]. In the general case, i.e., where the abovementioned condition may be not fulfilled, one should use iterative solution procedures of static problems, also, not always static problems can be solved simply, since they can be of rather large volume as well. If a static problem is non-linear, or the objective function is separable, then linear methods or that integer linear programming can be applied in the solution of static problems [4]. However, the latter methods are helpful only to solve problems of comparatively small volume.

The transport network considered usually has not so small number of elements (network nodes and arcs), and its objective function is not separable with respect to individual groups of variables. That is why, to solve static problems, decomposition principles are applied in turn. One of the possible ways of decomposition is usage of the linearization method [4]. Since some variables of the static problem, i.e., the variables that express the states of network elements are discrete variables, this problem acquires a combinatorial character. In order to solve problems whose variables naturally fall into discrete ones that determine the state of network elements and continuous ones that express the flow size in the network, there is a Benders decomposition procedure [5] that was formulated for solving linear problems, or its generalizations [6]. In the general case, the Benders decomposition method is an iterative procedure, using the optimization according to separate groups of variables and consisting of two stages. In the first stage, we optimize the flow distribution in the network under fixed technical states of its elements. In the second stage, we find optimal states of transport elements for the flow distribution found. The second stage of algorithm is formulated as a discrete optimization problem that is efficiently solved if there is only one limited resource. In other cases, one will have to use general discrete programming algorithms that are not so efficient.

Therefore we propose another, more universal way of solution. This paper discusses a method for solving the problem of transport network development, using the synthesis of a static section method and that of contour optimization developed by the author [7].

\section{Statement of the problem}

To formulate the problem of transport network development one has to be familiar with the topology of transport network in the shape of an oriented or non-oriented graph, its initial state, i.e., technical economic characteristics of its elements represented as arcs of the graph, data on the transportation volume in the network, their structure, and variation dynamics in the considered period. One also has to be aware of the limit of resources necessary for the network extension. One has to determine transport flow volumes and states of network elements per each year of the given period possible within the limits of the available resources, so that the summary discounted expenses for transportation and network development, were the least at the beginning of the considered period.

A model of the problem can be written as optimization model. The objective function of this model is a function, that expresses the dependence of network 
expenditure, including both the maintenance, expenditure and that on its development, on the flow volume in the network and its state. The constraints of the problem includes the flow continuity conditions, that relate the network topology, the transport flows and the volume of transportations and recourse limitation. Apart from the flow continuity conditions and resource limitation, the model includes a conditions that technical states of the transport networks elements can only increase. That is a natural condition since the transport objects are usually not destroyed or demolished, even with a considerable decrease in traffic intensity. The variables and parameters, that are contained by the objective function, and constraints of the model, i.e., transport flow sizes, the vector of transport network state, generalized incidence matrix, the vector of transportation volumes, amounts of resources and their limits, necessary for the network development and transportations are time functions. Incidence matrices describing the network may differ at certain time moments, because the transport network can be extended by building new elements of the network (e.g. by building new auto roads). The graph corresponding to the transport network represents not only existing, but also designed, i.e., alternative objects. Alternative objects can be built or not, dependent on the calculation results. Possible technical states of the transport element are determined by discrete variable integer values of that increase with an increase in the technical state of this element (e.g. the technical state of a motor-car road can be defined by the number of its traffic lines). The volume of resources for the reconstruction of the transport element changes jump like with a change in the state of the transport element (that changes in the same way). The solution to problem shows, which transport elements and at what moment have to be reconstructed as well as optimal transport flows for each year of the considered period.

A formal model of the problem can be written as follows

$$
\begin{gathered}
\min _{\left\{X^{t}, C^{t}\right\}} \sum_{t}^{T} \frac{F^{t}\left(X^{t}, C^{t}\right)}{(1+E)^{t}}, \\
S X^{t}=B^{t}, t=1, \ldots, T, \\
C^{\min } \leq C^{t} \leq C^{\max }, t=1, \ldots, T, \\
C^{t} \geq C^{t-1}, t=1, \ldots, T, C^{0}=C^{\min }, \\
R^{t}\left(C^{t}\right) \in \boldsymbol{R} .
\end{gathered}
$$

Here $t$ is the index of the current year; $T$ is duration of the considered period in years; $X^{t}=\left(x_{1}^{t}, \ldots, x_{L}^{t}\right)$ is the vector of product flow transported in the network; $x_{I}^{t}=\left(x_{i 1}^{t}, \ldots, x_{i n}^{t}\right)$ is the vector of product volume transported via the $i$-th transport element; $x_{i j}^{t}$ is the quantity of the $i$-th transport element load with the $j$-th product; $L$ is the number of transport network elements (arcs); $n$ is the number of products sorts transported; $C^{t}=\left(c_{1}^{t}, \ldots, c_{L}^{t}\right)$ is the vector that defines the transport network state; $c_{i}^{t}$ is a discrete quantity characterizing the technical state of the $i$-th transport element; $F^{t}\left(X^{t}, C^{t}\right)=\sum_{i}^{L} F_{i}^{t}\left(x_{i}^{t}, c_{i}^{t}\right)$ is a function that expresses the dependence of network expenses, including both the maintenance expenses and that on its development, on the flow volume in the network and its state; $F_{i}^{t}\left(x_{i}^{t}, c_{i}^{t}\right)$ is the cost function of the $i$-th transport element; $(1+E)^{t}$ is the coefficient of discounting; $S$ is a quasidiagonal matrix in the main diagonal of which there are node-arc incidence matrices for separate products of the given network and everywhere else there are zeros; $B^{t}$ is the vector of network node loading that defines the volume and structure of transportations; $R^{t}\left(C^{t}\right)$ is the vector of volumes of all kinds of resources necessary for changing the network state from state $C^{t-1}$ into state $C^{t} ; R$ is the set of resource limitation that is made more concrete when formulating the problem.

The cost function of an individual transport elements is the sum of the maintenance and capital expenses (pre-sented in comparison) which in turn are functions of loading and the technical state of this element, i.e.

$$
F_{i}^{t}\left(x_{i}^{t}, c_{i}^{t}\right)=f_{i}^{t}\left(x_{i}^{t}, c_{i}^{t}\right)+E \cdot K_{i}^{t}\left(c_{i}^{t}\right),
$$

where $f_{i}^{t}\left(x_{i}^{t}, c_{i}^{t}\right)$ is the function of maintenance expenses; $E$ is the standard efficiency coefficient of capital investments; $K_{i}^{t}\left(c_{i}^{t}\right)$ is the quantity of capital investments needed for building the $i$-th network element with appropriate technical-economic characteristics. Under the fixed technical state of a network element, the function of maintenance expenses can be expressed rather exactly as a convex function of the load level $x_{i}$ of this element. Besides, the maintenance expenses usually grow slower if the technical level of the element is higher.

Equality (2) expresses flow continuity conditions that 
relate the network topology with its arc and node loads. The sign of inequality between the vectors in conditions (3) and (4) denotes that this inequality is satisfied for all the coordinates of vectors. Condition (4) means that the states of transport network elements can increase only. This condition is rather natural since the transport objects built are usually not destroyed even with a very low traffic intensity via them. The solution to problem (1)-(5) is a vector $\left(X^{1}, C^{1}, \ldots, X^{T}, C^{T}\right)$ showing which transport elements and at what moment have to be reconstructed as well as optimal transport flows for each year of the con-sidered period.

\section{The method for problem solving}

The problem of long-term transport network development is not only problem characterized by great volumes, non-linear relations, but it is a non-linear problem with a lot of extreme. Therefore, one can expect to find only an approximate solution, close to a global one, at best. This paper discusses a method for solving the problems using the synthesis of static sections and contour optimization methods. The static section method allows us to replace a dynamic problem by a finite set of static problems, for-mulated for each year of the considered period. It allows us to decompose the initial problems into problems of a smaller volume, too. The way of problem solution depends on how the resource limitation is formulated in the model of problem.

If the resource limits are set for each year separately, i.e., constraints of problem (5) are concretized as follows:

$$
\sum_{i \in \mathbf{I}_{e}^{t}} R_{i}^{t}\left(c_{i}^{t}\right) \leq R^{t}, t=1, \ldots, T,
$$

where $\mathbf{I}_{c}^{t}$ is the set of numbers of the transport network elements reconstructed, i.e., for which $c_{i}^{t}>c_{1}^{t-1} ; R_{i}^{t}\left(c_{i}^{t}\right)$ is the vector of volumes of all kinds of recourses necessary for changing the $i$-the element of the network state from state $c_{i}^{t-1}$ into state $c_{i}^{t} ; R^{t}$ is the vector of resance limitation in the $t$-th year, then such a sequence of static problems is solved by a direct recursion method starting from the first year of the considered period:

$$
\min _{X^{t}, C^{t}} F^{t}\left(X^{t}, C^{t}\right)
$$

$$
\begin{gathered}
S X^{t}=B^{t}, \\
C^{t-1} \leq C^{t} \leq C^{\max }, C^{0}=C^{\min }, \\
\sum_{i \in \mathbf{I}_{c}^{t}} R_{i}^{t}\left(c_{i}^{t}\right) \leq R^{t},
\end{gathered}
$$

for each $t=1, \ldots, T$.

For the first, parameter values that determine the minimal states of the network also determine the state of the net-work, while the solution to the static problem solved determines the initial states of each new static problem of the sequence. The expense and resource functions are formed, respectively, taking account of possible alternation of the state of each network element from the initial level selected in this way up to the maximally possible one. Since each static problem is a non-convex one as shown below, there is no guarantee that the vector $\left(X^{t}, C^{t}\right)$ obtained by any known method is the solution to a static problem. Therefore, the general vector $\left\{X^{t}, C^{t}, t=1, \ldots, T\right\}$ obtained after solving the sequence of static problems (8)-(11) is usually not optimal nut rather a feasible, rational enough solution to considered problem (1)-(4), (6).

If the limits for all kinds of resources are set for the whole period considered without dividing them by a separate year, i.e., constraints (5) are concretized in the following way

$$
\sum_{t}^{T} \sum_{i \in \mathbf{I}_{c}^{t}} R_{i}^{t}\left(c_{i}^{t}\right) \leq R,
$$

then the dynamic problem is decomposed again into a sequence of static problems, in this case, however, this sequence is solved in inverse order. The firs problem of the sequence is formulated for the last year of a given period as follows

$$
\begin{gathered}
\min _{X^{T}, C^{T}} F^{T}\left(X^{T} C^{T}\right), \\
S X^{T}=B^{T}, \\
C^{\min } \leq C^{T} \leq C^{\max }, \\
\sum_{i \in \mathbf{I}_{C}^{T}} R_{i}^{T}\left(c_{i}^{T}\right) \leq R .
\end{gathered}
$$

The expense and resource functions here are formed en-visaging the variation of states of each transport element from the existing state up to the maximally admissible one. Having solved this static problem, we find final state of the network. We also establish thereby which network elements are to be recons- 
tructed as well as their final states. Time moments at which these elements are to be reconstructed are defined when solving the remaining problems of sequence

$$
\begin{gathered}
\min _{X^{t}, C^{t}} F^{t}\left(X^{t} C^{t}\right), \\
S X^{t}=B^{t}, \\
C^{\mathrm{min}} \leq C^{t} \leq C^{t+1},
\end{gathered}
$$

for all $t=T-1, T-2, \ldots, 1$. For each problem of the sequence, the states of transport elements are bounded from above, by forming the expense and the resource functions, respectively. The each static problem of sequence includes the flow continuity condition and other constraints described above. These static problems are solved by the method of contour optimization described below. The moment of reconstruction for each element to be reconstructed is determined as follows. For all the states $c_{i}^{t}>c_{t}^{\min }$ of the $i$-th transport element we fix time intervals $\left[t_{1}, t_{2}\right]$, in which the state does not change. The lower bound $t_{1}$ of this interval is just the moment of reconstruction of the $i$-th element into the state $c_{i}^{t}$.

Static problem can be treated as the problem of flow distribution in the transport network when technical states of the network elements can be changed. The static problem, however, qualitatively differs from the one as the state of network does not change. In this case, the dependence of costs of the volume of transportation will not be convex and the flow distribution problem itself becomes a multiextremal. The flow distribution can be described, for this case, by a non-linear model that includes both continuous and discrete variables. We propose this problem to solve in such a way. At first a non-linear discrete problem is modified by eliminating discrete variables. After eliminating discrete variables from the objective function and constraints, we replace the initial static problem by a non-linear programming with continuous variables.

Let us consider a case where the limits of resources are determined for each year separately of the considered period. We write the conditions of problem (8)-(10) as follows (for simplicity, we omit the index $t$ )

$$
\begin{gathered}
\min _{X, C} F(X, C)=\min _{X \in \mathbf{X}} \min _{C \in \mathbf{C}} F(X, C)= \\
\min _{X \in \mathbf{X}}\left(\sum_{i}^{L} \min _{c_{i} \in \mathbf{C}_{i}} F_{i}\left(x_{i}, c_{i}\right)\right)=
\end{gathered}
$$

$$
\min _{X \in \mathbf{X}} \sum_{i}^{L} F_{i}^{\min }\left(x_{i}\right)=\min _{X \in \mathbf{X}} F^{\min }(X)
$$

where $X$ is the set of feasible, i.e., satisfying conditions (9), flows; $C$ is the set of possible network states; $C i$ is the set of possible states of the $i$-th network element; $F_{i}^{\text {min }}\left(x_{i}\right)$ is a function that expresses the dependence of summary expenses of the $i$-th transport element on its load vector $x_{i}$ under optimal states of the element. This function can be regarded as an operator that finds such a value of the parameter ci at which we isolate a function with the lowest value out of a family of functions

$$
\left\{F_{i}\left(x_{i}, c_{i}\right), c_{i}^{\min } \leq c_{i} \leq c_{i}^{\max }\right\}
$$

under the given load $x_{i}$ of the element. We can represent the function $F_{i}^{\mathrm{min}}\left(x_{i}\right)$ graphically as a curve covered from below of the family of functions (21). For alternative elements it is discontinuous at the zero load point.

We assume that in the case of reconstruction, both the technical state of an element and the quantity of the resources utilized change jump like (in the opposite case, we were not able to decompose a dynamic problem into a sequence of static problems). Thus, the vector $R_{i}\left(c_{i}\right)$ of resource quantity utilized is a vector function of variables $x_{i}$, i.e.,

$$
R_{i}\left(c_{i}\right)=R_{i}\left(c_{i}\left(x_{i}\right)\right)=\tilde{R}_{i}\left(x_{i}\right),
$$

where dependence $\tilde{R}_{i}\left(x_{i}\right)$ is a step like function of the element load $x_{i}$.

Consequently, we can replace each static problem with discrete-continuous variables after eliminating the discrete variables $c_{i}$ by non-linear a programming problem

$$
\begin{gathered}
\min _{X \in X} F^{\min }(X), \\
\sum_{i}^{L} \tilde{R}_{i}\left(x_{i}\right) \leq R .
\end{gathered}
$$

The dependence of transportation expense, technical state of network element, consumed resource and the shape of the function $F^{\min }(X)$ for one-dimensional care are illustrated in Fig 1.

This problem is a non-convex one and includes a conditions of resource limitation only. The idea of a penalty function enables us to avoid constraints of 

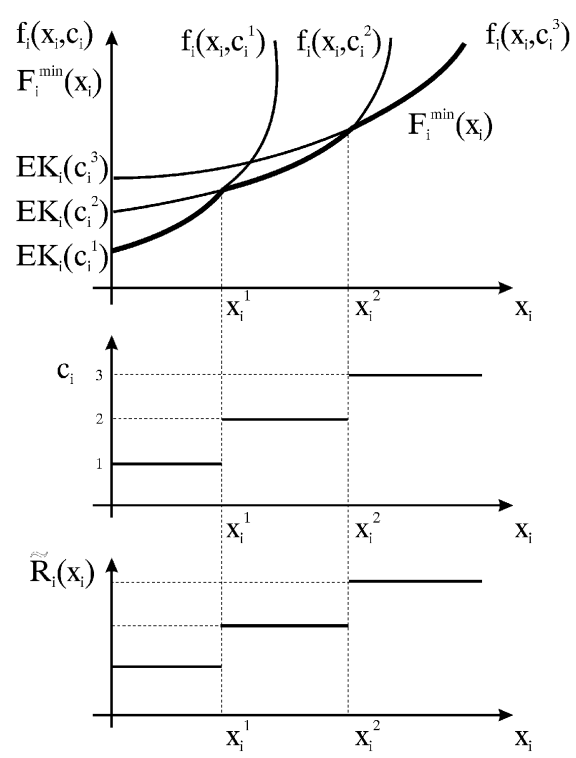

Fig 1. Dependence of discounted expense, technical state and consumed resource amount on the exted of loading of transport element

resource limitation in direct form by including that into the objective function of the problem. The nonlinear problem itself is replaced by sequence of problems with the objective function modified in this way. Solution of such a problem is problematic due to the fact the penalty function is not only non-convex, but also non-smooth and discon-tinuous and there are no methods to effectively solve it even in the sense of a local solution. Since smoothness of the penalty function in this case is violated on the sets of points described by linear equations that we call critical equations, then we can successfully use the contour op-timization method to solve the problem, because it disre-gard the indicated properties of the function minimized.

The method of contour optimization is based on the idea of modified coordinate descent and properties of graphs that associate the structures of graphs and systems of equations describing the flow distribution in the network. The algorithm of contour optimization is an iterative procedure at each iteration of which the distribution of flow of only one product in the network is optimized, the distribution of all the other products being invariant. An iteration of algorithm consists of the one - dimensional minimization procedure of the summary expense function of contour cyclically repeated for each contour. Special graph structure alternating procedure is used to accelerate the convergence of the algorithm after flow optimization in each contour in the network.
An iteration of algorithm is formally written as follows

$$
\min _{a \leq \Delta x_{k}^{j} \leq b} F_{k}^{j}\left(\Delta x_{k}^{j}\right)=\sum_{i \in I_{k}^{j}} f_{i}\left(x_{i} \mathrm{II} x_{i}^{j}+e_{i k}^{j} \Delta_{k}^{j},\right.
$$

here $j$ is the number of the product distributed in a given iteration; $k$ is the number of a free arc that formed a contour by joining it to the tree; $x_{i}^{j}$ is the $i$-th arc load with the $j$-th product; $\Delta x_{k}^{j}$ is a change in the free arc load with the $j$-th product; $\mathbf{I}_{k}^{j}$ is the set of numbers of graph arcs that belong to the contour formed by the $k$-th arc; $e_{i k}^{j}$ is a coefficient that is equal to +1 if the direction of $I$-th and $k$-th arc in the contour are the same, and to -1 in the opposite case.

The optimization interval $[a, b]$ is the smallest change interval of the free arc load with the $j$-th product, in which zero load with this product of any arc of the contour is obtained.

If the flow $X$ obtained by the algorithm described, satisfies one or several critical equations that are written as a system of equations

$$
y=A x+A^{0},
$$

there is no guarantee that this flow distribution corresponds to the local solution of problem found with a certain accuracy. In this case, we can go over to the second stage of algorithm after rearranging beforehand the system of equations (26) into another shape

$$
\bar{x}=\bar{A}_{z}+\bar{A}^{0},
$$

where the coordinates of basic variable vector $\bar{x}$ are part of independent variables of the system of equations (26), that is solved with respect to independent variables, while the rest independent variables of this system are the coordinates' of vector $z$.

The second stage of algorithm is an iterative procedure that cyclically repeats one-dimensional optimization for each independent variable $z_{k}=x_{i}^{j}$, until the stopping condition of algorithm is satisfied. In this case, we minimize the summary expense function of arcs of not a single but of several contours called connected contours, whose loads change with a change in the value of $z_{k}$. The system of equations (27) shows which arc loads of the contours will change and in what way will they change. The optimization 
procedure for the second stage of algorithm is written as follows

$$
\min _{a \leq z_{k} \leq b} F\left(\Delta z_{k}\right)=\sum_{l \in L_{k}} f_{l}\left(x_{l}\left(\Delta z_{k}\right)\right)
$$

where $[a, b]$ is the minimal interval of changes $\Delta z_{k}$ of the independent variable $z_{k}$, in which we obtain zero load of any arc of the connected contours with the product whose flow varies in this arc with the variation of $z_{k} ; L_{k}$ is the set of arcs of all the connected contours. If after procedure (28) the flow satisfies an additional critical equation, then we supplement matrix $\bar{A}$ of the system of equations (27) with the coefficients of this equation expressed with independent variables. Afterwards we rearrange the vectors $\bar{x}$ and $z$ by supplementing the vector $\bar{x}$ of basic variables with the independent variable $z_{k}=x_{i}^{j}$ removing it from independent variables and by recalculating the elements of matrix $\bar{A}$, respectively.

In this case, we minimize the summary expense function of arcs of the network of not a single but of several contours called connected contours, whose flows change with a change in the value of the independent variable of problem. In each connected contour changes flow of only one product but in a separate contour changes flow of different products. In the second stage of algorithms the multicommodity transport flow is optimized in the set of points defined by a system of the critical equations that satisfies the transport flow.

The algorithm of contour optimization is rather efficient in terms of calculation since for one dimensional optimization we can use some of very efficient procedures of univariate optimization. On the other hand, data needed for solving the problem can be written in the most compact way using the means of graphs. Contour optimization method, however, like any other known optimization method converges only to the local solution. To improve the solution it is reasonable to use the synthesis of random search and contour optimization methods, i.e., to optimize using a number of randomly generated points.

\section{Calculating experiment}

Under the abovementioned conditions that are contained in constraints of the optimal network development problem under consideration, a dynamic problem can be replaced by a finite sequence of static problems. Therefore if suffices to experimentally define the usability of the method proposed for solving a static network development problem, as the parameters characterizing its technical state can be varying. Also, this static problem is not convex, therefore the known methods can help find only its approximate solution without a guarantee of finding the optimal solution. Thus, there cannot exist a unique method, absolutely better than all others. The quality of a solution obtained by some method will depend on par-ticular conditions, i.e., under certain conditions one method can be better than another, under other condi-tions. To determine these conditions is also not always a solvable problem. In any case, however, the method should ensure a sufficiently good solution. In order to define the usability of the contour optimization method for solving network development problems of practical importance, an experiment by calculation has been carried out. The method has been tested, by using a network that was a part of the real railway network of the country at the time, as well as the data described in [3]. The network, illustrated in Fig 2, consists of 43 transport points und 49 railway lines, connecting these points, 27 of which are oneway, represented by a thin line, and 22 two-way lines, represented by a thick line. The numbers over the lines that represent railway routes denote their length in kilometres.

Table presents cargo volumes (correspondences) in thousands of tons that are sent from the departure points to their destination. The departure points are indicated in the rows of the table, and destination points - in the columns.

Transportation expenses by railway depend on the load size of a line (i.e., transported cargo volume) and its technical level, that determines the type of line. Three railway line types are usually distinguished: a one-way

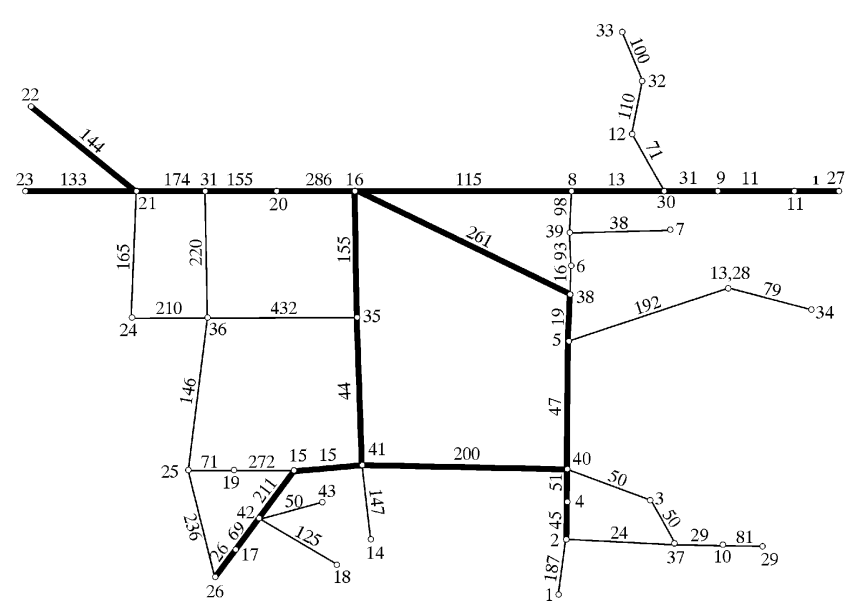

Fig 2. Discussed transport network and its initial technical state 
Matrix of transportation for discussed transport network

\begin{tabular}{|c|c|c|c|c|c|c|c|c|c|c|c|c|c|c|c|c|c|c|c|c|c|c|c|c|c|c|c|c|}
\hline Nodes & 1 & 2 & 3 & 4 & 5 & 6 & 7 & 8 & 9 & 10 & 11 & 12 & 13 & 14 & 15 & 16 & 17 & 18 & 19 & 20 & 21 & 22 & 23 & 24 & 25 & 26 & 27 & 28 \\
\hline 1 & & 660 & 40 & 30 & & & 7 & & & & 7 & & & & 3 & 4 & 2 & & 5 & 5 & 25 & 12 & 12 & 9 & 2 & & & 8 \\
\hline 2 & 150 & & & 24 & 12 & 15 & 40 & 15 & 10 & 22 & 50 & 25 & & 40 & 50 & 75 & 15 & 7 & 15 & 35 & 25 & 35 & 25 & 30 & 12 & & 75 & 430 \\
\hline 3 & 20 & & & 20 & 10 & & 25 & 7 & & 12 & 30 & 20 & & 25 & 40 & 65 & 15 & & 44 & 30 & 25 & 30 & 25 & 25 & 10 & & 60 & 120 \\
\hline 4 & 30 & 400 & 40 & & & 7 & 200 & 5 & & 7 & 30 & & 10 & 80 & 120 & & 120 & & & & \begin{tabular}{|l|}
110 \\
\end{tabular} & 400 & 450 & 300 & 220 & & 30 & 40 \\
\hline 5 & 7 & 250 & 230 & & & & 7 & & & 7 & 30 & & & & 130 & 240 & & 40 & & 40 & 300 & 300 & 200 & 340 & 250 & & 30 & 30 \\
\hline 6 & 15 & 15 & & 7 & & & 7 & & 5 & 7 & 30 & & & & 8 & 350 & & & 130 & 80 & 600 & 600 & & 10 & & 20 & & 15 \\
\hline 7 & 30 & 30 & 10 & 15 & 15 & 6 & -1 & 5 & 5 & 10 & 50 & 25 & 10 & 20 & 30 & 15 & 15 & 15 & 15 & 15 & 120 & 30 & 25 & 25 & 15 & 50 & & 40 \\
\hline 8 & 20 & 15 & 7 & 7 & & 5 & 5 & & & & 30 & & & & 5 & 17 & 5 & & & 7 & 10 & & 8 & 8 & 5 & 30 & & \\
\hline 9 & 15 & 8 & 8 & & 7 & & 5 & 50 & & & & 500 & & & & 5 & & & & 5 & 20 & 30 & & & 30 & & & \\
\hline 11 & & 10 & 5 & & 7 & & 30 & 10 & 18 & & & 10 & & & 2 & 4 & & & & 4 & 15 & 15 & 15 & 12 & & 20 & & \\
\hline 12 & & 250 & 8 & 15 & 15 & 15 & 22 & 10 & 15 & & 8 & & & & 15 & 30 & 7 & 4 & & 9 & 55 & 60 & 15 & 15 & 40 & 20 & & \\
\hline 13 & & & & & & & & & & & & & & & & & & & & & & & & & & & 700 & \\
\hline 14 & & 2 & & 2 & & & 2 & & 2 & & 1 & 3 & & & 30 & 15 & 35 & 15 & 10 & 5 & 3 & 3 & 6 & 12 & \begin{tabular}{l|}
12 \\
\end{tabular} & & 6 & 20 \\
\hline 15 & 3 & 6 & & 4 & & 6 & 4 & & 6 & & 4 & 6 & & 50 & & 40 & 110 & 50 & 40 & 10 & 5 & 5 & 10 & 20 & 40 & & 12 & 50 \\
\hline \begin{tabular}{l|}
16 \\
\end{tabular} & 7 & 18 & 8 & 10 & 10 & $\overline{9}$ & 16 & $\overline{4}$ & & 6 & 20 & 24 & & 35 & 100 & & 20 & 8 & 170 & 20 & 100 & 100 & 35 & 35 & 20 & 130 & & 30 \\
\hline 17 & & 2 & & 2 & & & 2 & & 6 & & 2 & 2 & & 15 & 30 & 40 & & 20 & 6 & 2 & 7 & 8 & & 35 & 40 & & 30 & 60 \\
\hline 18 & & 1 & & 2 & & & 2 & 2 & & & 2 & 2 & & 8 & 25 & 15 & 8 & & 4 & & 8 & 8 & 3 & 20 & 20 & & 15 & 30 \\
\hline 19 & & 3 & & & 2 & & 3 & & & 2 & 1 & 8 & & $\pi$ & 3 & 69 & 80 & 6 & & 15 & 45 & 25 & & & 20 & & & \\
\hline 21 & 6 & 15 & 8 & 8 & 10 & & 15 & 15 & & & 20 & 35 & & 15 & 50 & 170 & 35 & 25 & 45 & 200 & & & & & 110 & 930 & & 50 \\
\hline 22 & 15 & 15 & 8 & 8 & & 10 & 15 & & 15 & & 25 & & & 15 & 60 & 200 & 40 & 25 & 50 & & & & 230 & & 110 & 930 & & 50 \\
\hline 23 & & 20 & & 20 & 6 & & 20 & & 7 & & 20 & 15 & & 15 & 40 & 40 & 30 & 15 & & & & & & & & & 380 & \\
\hline 24 & 10 & 20 & 10 & 15 & & & 15 & & 5 & 10 & 15 & 20 & & \begin{tabular}{|l|}
17 \\
\end{tabular} & 50 & 65 & 40 & 20 & & & & & & & 210 & & 150 & 320 \\
\hline 25 & 6 & 5 & & & & 7 & & & & 2 & 10 & 10 & & 30 & 75 & 60 & 100 & 60 & & 40 & 60 & 60 & & 450 & & & 50 & 120 \\
\hline 26 & & & & & & 7 & 15 & 15 & & & 50 & 120 & & & & 45 & & 8 & 9 & 25 & 330 & 410 & & & & & & \\
\hline 27 & & 20 & & 20 & & & & & & & & & & 7 & 12 & & 8 & & & & & & 310 & 200 & 300 & & & \\
\hline 28 & 6 & 350 & 500 & 15 & & 15 & & & & 15 & & 15 & & 8 & 12 & 15 & 10 & 7 & & 12 & 50 & 50 & & 420 & 550 & & & \\
\hline
\end{tabular}

railway line, one-way line with detours, and a two-way line. Each variant of these lines is described by the cost function per $1 \mathrm{~km}$. Expenses for the entire road are obtained by multiplying the costs per $1 \mathrm{~km}$. by the road length. In all cases, the values of the cost function depend on the cargo flow size in both directions of the road, i.e.,

$$
f_{i}^{\min }\left(x_{i}\right)=f_{i}\left(x_{i}^{+}, x_{i}^{-}\right),
$$

where $x_{i}^{+}$and $x_{i}^{-}$stand for cargo flow volumes in one and the other direction of the railway road. If we calculate the flow distribution by the contour optimization method, the railway route is represented in the shape of an oriented graph, and the size of cargo flows, following in the direction of an arc, is denoted by $x^{+}$, while in the opposite direction, - by $x^{-}$.

When calculating, we followed the conditions, determined in [3]. In that work, the shape of the cost function, that expresses the dependence of transportation expenses in an individual railway line on two quantities, is as follows:

$$
\bar{f}_{i}\left(v_{i}, w_{i}\right)=\left[\varphi_{i}^{1}\left(v_{i}\right)+\varphi_{i}^{2}\left(v_{i}\right) w_{i}\right] l_{i}
$$

here $v_{i}=\max \left(x_{i}^{+}, x_{i}^{-}\right), w_{i}=\min \left(x_{i}^{+}, x_{i}^{-}\right), l_{i}$ is the length of the $i$-th railway line. The functions $\varphi_{i}^{i}$ and $\varphi_{i}^{2}$ depend on the type of line. For a one-way railway line,

$$
\begin{gathered}
\varphi_{i}^{1}\left(v_{i}\right)=\frac{137,2 v_{\mathrm{i}}^{2}}{14,4-0,27 v_{\mathrm{i}}}+660 v_{i} \text { and } \\
\varphi_{i}^{2}\left(v_{i}\right)=\frac{52,8 v_{i}}{14,4-0,27 v_{i}}+130 .
\end{gathered}
$$

For a two-way railway line,

$$
\varphi_{i}^{1}\left(v_{i}\right)=660 v_{i} \text { and } \varphi_{i}^{2}\left(v_{i}\right)=130 .
$$

For a one-way railway line with detours,

$$
\varphi_{i}^{1}\left(v_{i}\right)=660 v_{i}+28,1\left(v_{i}-28\right)^{2} \cdot \max \left(v_{i}-28 ; 0\right)
$$

and $\varphi_{i}^{2}\left(v_{i}\right)=130$.

Detours in the one-way line allow trains to pass each other in the case of an increase in traffic intensity. The formula presented shows that, in the route with detours, the transportation expenses are calculated just like in the two-way line, if $v_{i} \leq 28$ (mil. tons/year). If the cargo flow in the direction of higher transportation intensity exceeds this quantity, then extra expenses arise in the route. In [3], it has been established that if the normative effectiveness value is $E=0,1$, the cost of construction of $1 \mathrm{~km}$ detour is 90 thous. rb, the cost of building $1 \mathrm{~km}$ of a second way is 180 thous. rb, then, in economic terms, it is reasonable to build detours, if the cargo volume transported by a one-way railway line in the direction of its higher loading exceeds 22,5 (mill. tons/year), or a second way, if the cargo flow exceeds 32 (mill. tons/year). 
In the initial version of the network under consideration there are only two types of railway lines: one-way railway lines and two-way lines. When optimizing the transport network state, it is necessary to envisage a possibility of reconstructing a one-way railway line into a line with detours, or a two-way railway line. In [3], the optimal state of the network considered has been determined using a version of the linearization method, called a consistent distribution method, in the scheme of Benders decompositions.

The linearization method makes it possible to define flow sizes in the network under fixed states of network elements. That is an iterative procedure, where in each iteration, network arc loads are decreased by removing a certain part of all the correspondences from the network, that afterwards are redistributed by the shortest routes, in the sense of differential expenses. Differential expenses are partial derivatives of the objective function multiplied by the respective arc lengths. Thus, differential expenses are the measure of length' of network arcs. Since a static network development problem was formulated without source constant restriction conditions, optimal states of network elements under the set cargo flow distribution are defined by a simple rule mentioned above. If the flow size in the route in the direction of maximal transportation route exceeds 22,5 mill. tons but is lesser than 32 mill. tons, then the one-way line should be reconstructed into a one-way line with detours. If the flow exceeds 32 mill. tons, then another way ought to be built in it. It must be admitted that the Benders decomposition and the method of consistent distribution are wide used in the solution of problems in practice and yield good results.

Since resources are not limited in the considered case, only one static problem was solved by the contour optimization method. The contour optimization algorithm was corrected a little in this experiment, an iteration of the algorithm was regarded as a single optimization in all the contours of cargo networks. The correction enabled us to approximate iteration structures of both algorithms to some extent and made it possible to compare them. The initial state of the network was set the same for both algorithms. After 50 iterations in each, the results obtained for both algorithms compared were similar. The value of the optimal criterion, obtained by means of Benders and consistent distribution algorithms is equal to 89,13 and by the contour optimization algorithm is 89,31 . Flow distribution in the network also differed but a little, by several fractions of per cent. One cannot derive any generalizations from these results, since the iteration structures of the algorithms compared differ to some extent. A more important fact is that qualitative results, obtained by both methods, were coincidental: the optimal state of the network under a given transportation volume is achieved by reconstructing one-way railway lines 5-13 and 6-38 into one-way lines with detours.

Despite that in the case of the problem with several extrema, the contour optimization method has certain advantages as it is not only a strictly local method the calculation results obtained for the considered problem are similar, maybe because the problem considered is unimodal, or the global minimum is expressed explicitly and has a wide zone of "attraction".

It is possible to estimate the algorithms universally only in the process of multiple solution of real problems, however, the calculation results for this particular case show that the contour optimization algorithm 'works' rather well and can be used for calculation in practice. This is the main conclusion of the experiment. However, some of the features of the algorithm are quite obvious. In the vicinity of the solution, first or second-order algorithms (the consistent distribution algorithm is attributed to the former) converge faster than the zero-order algorithms to which the proposed algorithm is attributed. On the other hand, since the practical data are not absolutely exact, it is hardly reasonable to strive for a higher order calculation accuracy than the error of data. Therefore, a decrease in the rate of convergence in the surroundings of the solution is not the essential shortcoming of the algorithm proposed. The consistent distribution algorithm consumes the largest share of calculation time to find the shortest routes and this share is relatively increasing with an increase of volume of the problem [3], meanwhile the calculation duration of the contour optimization algorithm is predetermined by the cost function. The more complex the objective function, the faster the calculation duration increases. This drawback can be avoided, by using a piecewise linear objective function approximation. It is worthwhile to iterate calculations by specifying ever more the objective function approximation in the vicinity of the solution. If we approximate nearly to the solution, the last cycle of calculations should be performed with the given objective functions. This way of arranging calculations would allow us to diminish the calculation time duration up to the agreeable quantity.

\section{Conclusions}

As the experimental calculations have shown, the proposed method, based on the synthesis of static 
sections and contour optimization methods, also applies to solve problems of practical significance. The method proposed has its own advantage. While the Benders decomposition is effective in the case, where there is only one recourse restriction, the contour optimization algorithm is effective for any number of resource restrictions. On the other hand, its effectiveness is not decreased by lack of smoothness and discontinuity of the objective function. Using the piecewise linear approximation of the objective function which allows us to diminish the calculation time, the proposed method also applies to solve problems of rather a great volume as well as in the case, where the transportation costs and variables of the problem are related by complex dependences.

\section{References}

1. Magnanti, T. L.; Wong, R. T. Network design and transportation planning models and algorithms. Transportation Science, 1984, 18(1), p. 3-55.

2. Minoux, M. Network synthesis and optimum network design problems: models, solution methods and applications. Networks, 1989, 19(2), p. 313-360.

3. Левит, Б. Ю.; Лифшиц, В. Н. Нелинейные транспортные задачи. Москва: Транспорт, 1972.

4. Kennington, J. A.; Helgasson, A. V. Algorithms for Network Programming. John Wiley ans Sons, New York, 1980.

5. Benders, L. F. Partitioning procedure for solving mixevariable programming problem. Numerishe Mathematik (Berlin), 1962, 4(3), p. 238-252.

6. Geofrion, A. M. Generalized Benders decomposition. Journal Optim. Theory and Appl., 1972, Vol 10, No 4, p. $237-260$.

7. Davulis, G. A method of contour optimization for transport flow distribution. Statistics in Transition, 1999, 4(2), p. 201-212. 